EXTENDED REPORT

\title{
Optimised, low cost, low field dedicated extremity MRI is highly specific and sensitive for synovitis and bone erosions in rheumatoid arthritis wrist and finger joints: comparison with conventional high field MRI and radiography
}

\author{
B J Ejbjerg, E Narvestad, S Jacobsen, H S Thomsen, M Østergaard
}

Ann Rheum Dis 2005;64:1280-1287. doi: 10.1136/ard.2004.029850

See end of article for authors' affiliations

Correspondence to:

Dr B J Ejbjerg, Department of Rheumatology,

Copenhagen University

Hospital at Hvidovre,

Kettegaard Allé 30, 2650

Hvidovre, Denmark;

ejbjerg@dadlnet.dk

Accepted 2 January 2005

Published Online First

13 January 2005
Objective: To evaluate a low field dedicated extremity MRI unit for detection of bone erosions, synovitis, and bone marrow oedema in wrist and metacarpophalangeal (MCP) joints, with a high field MRI unit as the standard reference.

Methods: In 37 patients with RA and 28 healthy controls MRI of the wrist and 2nd-5th MCP joints was performed on a low field MRI unit (0.2 T Esaote Artoscan) and a high field MRI unit (1.0 T Siemens Impact) on 2 subsequent days. MRI was performed and evaluated according to OMERACT recommendations. Additionally, conventional $x$ ray, clinical, and biochemical examinations were performed. In an initial low field MRI "sequence selection phase", based on a subset of 10 patients and 10 controls, sequences for comparison with high field MRI were selected.

Results: With high field, spin echo MRI considered as the reference method, the sensitivity, specificity, and accuracy of low field 3D gradient echo MRI for erosions were $94 \%, 93 \%, 94 \%$, while the corresponding values for $x$ ray examination were $33 \%, 98 \%$, and $83 \%$. Sensitivity, specificity, and accuracy of low field MRI for synovitis were $90 \%, 96 \%$, and $94 \%$, and for bone marrow oedema $39 \%, 99 \%$, and $95 \%$. Intraclass correlation coefficients between low field and high field scores were $0.936(p<0.005)$ for bone erosions and $0.923(p<0.05)$ for synovitis.

Conclusion: Low field MRI provides high accuracy for detection and grading of erosions and synovitis, with high field MRI as the standard reference. For bone marrow oedema, specificity is high, but sensitivity only moderate. Low cost, patient compliant, low field dedicated extremity MRI provides similar information on bone erosions and synovitis as expensive high field MRI units.
$\mathrm{T}$ he treatment strategy in rheumatoid arthritis (RA) has changed markedly during the past decade with emphasis on completely suppressing joint inflammation and preventing structural joint damage and functional disability as early as possible during the course of the disease. ${ }^{1-3}$ Owing to this early and extensive treatment approach, there is a growing need for sensitive and specific tools for both early diagnosis and monitoring disease activity and joint damage. Furthermore, markers of prognostic significance are also needed.

The examination modalities currently used for assessment of disease activity and structural joint damage-clinical examination, composite disease activity scores, biochemical assessment, and conventional radiography-are not sufficiently sensitive or specific, especially in early disease. ${ }^{4-6}$

In recent years new methods for diagnosing and monitoring RA have evolved. In particular, magnetic resonance imaging (MRI) is a promising tool as it offers the opportunity for an examination of all aspects of the rheumatoid joint disease, visualising both destructive and inflammatory disease manifestations.

MRI performed on high field units has in previous studies been more sensitive than conventional methods for detection of synovitis and bone changes in patients with RA. ${ }^{\text {7-10 }}$

Low field dedicated extremity MRI units are more comfortable for the patients and less expensive, but, largely unvalidated. ${ }^{11-14}$
The objective of this study was to evaluate a low field dedicated extremity MRI unit for the detection and grading of synovitis, bone marrow oedema, and MRI erosions in wrist and metacarpophalangeal (MCP) joints, with a high field MRI unit as the standard reference, and to compare the MRI findings with the destructive joint changes seen on $x$ ray examination.

\section{PATIENTS AND METHODS}

Thirty seven patients ( 13 men, 24 women) with RA, fulfilling the American College of Rheumatology 1987 revised diagnostic criteria ${ }^{15}$ and 28 healthy controls ( 8 men, 20 women) were enrolled in the study. All subjects were recruited from the Department of Rheumatology, Copenhagen University Hospital at Hvidovre. Local ethics committee approval was obtained before starting the studies.

\section{Clinical and biochemical examination}

All clinical examinations were performed by the same rheumatologist and included assessment of joint swelling and joint tenderness as recommended by EULAR. ${ }^{16}$ Furthermore, every person taking part in this study

Abbreviations: FOV, field of view; FS, fat saturated; GE, gradient echo; ICC, intraclass correlation coefficient; MCP, metacarpophalangeal; MRI, magnetic resonance imaging; $R A$, rheumatoid arthritis; $S E$, spin echo; STIR, short $\tau$ inversion recovery; TE, echo time; TR, repetition time 
completed a Health Assessment Questionnaire. ${ }^{17}$ The laboratory tests covered serum $\mathrm{C}$ reactive protein (reflection photometry) and IgM rheumatoid factor (turbidometry). Disease Activity Scores based on 28 joint assessments were computed for all patients. ${ }^{18}$

\section{Conventional radiography}

An $x$ ray examination of the wrists and hands was performed in the posteroanterior and oblique projections. ${ }^{19}$ All radiographs were evaluated by the same radiologist, who had no access to data and results of the MRI findings. Radiographic bone erosions were evaluated separately in each wrist bone and MCP bone quadrants.

\section{Magnetic resonance imaging}

MRI of the wrist and 2nd-5th MCP joints of the dominant hand was performed twice on two different MRI units-that is, a 1.0 T Siemens Impact high field MRI unit equipped with a circular polarised transmit-receive coil and a $0.2 \mathrm{~T}$ Artoscan (Esaote Biomedica) low field dedicated extremity MRI unit equipped with a dual phased array coil.

The MRI scans were performed on two subsequent days to ensure complete clearance of the intravenously injected contrast agent $(0.1 \mathrm{mmol}$ gadolinium-DTPA-BMA $/ \mathrm{kg}$ body weight; Omniscan (Amersham Health)) and still allowing only minimal time for biological variations.

\section{High field MRI}

On the high field MRI unit, the following procedures were performed: the subjects were positioned prone with the hand fixed above the head in the centre of the coil (superman position). $\mathrm{T}_{1}$ weighted, spin echo $\left(\mathrm{T}_{1}-\mathrm{SE}\right)$ sequences were obtained in the coronal and axial planes before and after intravenously injected contrast medium (Omniscan). In addition, coronal short $\tau$ inversion recovery (STIR) and coronal $\mathrm{T}_{2}$ weighted, spin echo, fat saturated $\left(\mathrm{T}_{2}\right.$-SE-FS) sequences were obtained in the coronal plane before the contrast agent was administered.

The imaging variables for the different MRI sequences were as follows: $\mathrm{T}_{1}$-SE images: repetition time (TR) $600 \mathrm{~ms}$,

Table 1 Characteristics of patients and healthy controls

\begin{tabular}{lll}
\hline Characteristics & $\begin{array}{l}\text { Controls } \\
\text { (n=28) }\end{array}$ & $\begin{array}{l}\text { Patients } \\
\text { (n=37) }\end{array}$ \\
\hline Age (years) & $38(24-57)^{*}$ & $52(33-84)^{*}$ \\
Sex (female/male) & $20 / 8$ & $24 / 13$ \\
Disease duration (years) & $0(0-0)^{*}$ & $5(1-37)^{*}$ \\
Tender joints (0-28) & $0(0-0)^{*}$ & $8(0-22)^{*}$ \\
Swollen joints (0-28) & $0(0-0)^{*}$ & $6(0-16)^{*}$ \\
Morning joint stiffness (min) & $0(0-0)$ & $15(0-120)$ \\
HAQ (0-3) & $0(0-0)^{*}$ & $0.875(0-2.38)^{*}$ \\
s-CRP (ref. <10 mg/l) & $8(8-16)^{*}$ & $10(8-111)^{*}$ \\
IgM-RF (ref <17 kIU/l) & $<10(<10-31)^{*} 123(<10-4220)^{*}$ \\
IgM-RF positivity & $3.5 \%$ & $73 \%$ \\
DAS28 (serum CRP based) & NA & $4.36(2.0-6.9)$ \\
Disease activity & & \\
High (>5.1) & - & 9 \\
Intermediate (3.2-5.1) & - & 23 \\
Low (2.6-3.2) & - & 0 \\
Clinical remission (<2.6) & - & 70 \\
Drugs (\%) & & 13 \\
DMARDs & - & 16 \\
Targeted biological therapy & - & 29 \\
Corticosteroids & - & \\
NSAIDs & $\neq$ & \\
Analgesics & $\neq$ & \\
\hline
\end{tabular}

*Values are medians (ranges) unless otherwise stated. $H A Q$, Health Assessment Questionnaire; s-CRP, serum $C$ reactive protein; IgM-RF, IgM rheumatoid factor; DAS28, Disease Activity Score based on 28 joints' assessment; DMARDs, disease modifying antirheumatic drugs; NSAIDs, non-steroidal anti-inflammatory drugs; $\neq$, occasional use due to headache, for example. echo time (TE) $15 \mathrm{~ms}$, slice thickness $3 \mathrm{~mm}$, field of view (FOV) $109 \mathrm{~mm} \times 145 \mathrm{~mm}$, matrix $192 \times 256$, number of acquisitions 2, number of repetitions 1; STIR sequence: TR $4500 \mathrm{~ms}$, TE $30 \mathrm{~ms}$, inversion time $150 \mathrm{~ms}$, slice thickness $3 \mathrm{~mm}$, FOV $109 \mathrm{~mm} \times 145 \mathrm{~mm}$, matrix $182 \times 256$, number of acquisitions 3, number of repetitions $1 ; \mathrm{T}_{2}$-SE-FS sequence: TR $4500 \mathrm{~ms}$, TE $96 \mathrm{~ms}$, slice thickness $3 \mathrm{~mm}$, FOV $109 \mathrm{~mm} \times 145 \mathrm{~mm}$, matrix $182 \times 256$, number of acquisitions 3, number of repetitions 1 .

\section{Low field MRI}

On the low field MRI unit the following procedures were performed: the subjects were seated in an adjustable chair with the dominant hand positioned in the centre of the coil in a neutral position. $\mathrm{T}_{1}$-SE sequences were obtained in the coronal and axial planes supplemented with a $\mathrm{T}_{1}$ weighted three dimensional gradient echo $\left(\mathrm{T}_{1}-\mathrm{GE}\right)$, with isometric voxels, before and after intravenously injected contrast medium (Omniscan). The gradient echo images were subsequently reconstructed with a slice thickness of $1 \mathrm{~mm}$. A coronal STIR sequence was obtained before the contrast agent was administered.

The imaging parameters for the different MRI sequences were as follows: $\mathrm{T}_{1}$-SE images: TR $550 \mathrm{~ms}$, TE $18 \mathrm{~ms}$, slice thickness $3 \mathrm{~mm}$, FOV $200 \mathrm{~mm} \times 200 \mathrm{~mm}$, matrix $256 \times 256$, number of acquisitions 3; 3D-GE sequence: TR $30 \mathrm{ms,}$ TE $12 \mathrm{~ms}$, slice thickness $1 \mathrm{~mm}$, FOV $140 \mathrm{~mm} \times$ $140 \mathrm{~mm} \times 80 \mathrm{~mm}$, matrix $192 \times 160 \times 80$, number of acquisitions l, flip angle $65^{\circ}$; STIR: TR $1100 \mathrm{~ms}$, TE $24 \mathrm{~ms}$, inversion time $85 \mathrm{~ms}$, slice thickness $3 \mathrm{~mm}$, FOV $200 \mathrm{~mm}$ $\times 200 \mathrm{~mm}$, matrix $192 \times 160$, number of acquisitions 3 , interslice gap $0.3 \mathrm{~mm}$.

\section{MRI interpretation: preliminary evaluation}

To compare the sensitivity and specificity of the 3D gradient echo (recalled images) and the 2D spin echo sequence of the low field MRI unit, we initially evaluated MR images from 10 patients and 10 healthy subjects. Conventional high field MRI was considered the standard reference.

At the time of the preliminary MR image analysis there was no internationally accepted scoring system for MR images in RA, as later developed by the OMERACT MRI in RA Group. ${ }^{20}$ Accordingly, the scoring in the preliminary MR image evaluation was based on the methods used by our group in previous studies. ${ }^{21} 22$

Each image set (low field spin echo, low field gradient echo, and high field spin echo) was evaluated in a blinded setting for the presence or absence of MRI erosions and synovitis. The evaluation of bone erosions comprised separate assessment of each MCP quadrant and wrist bone, and the evaluation of synovitis covered assessment of each joint. ${ }^{23}$ In view of the results of the preliminary evaluation (see later), we chose to use the 3D-GE recalled images from the low field unit for the subsequent main MR image evaluation.

In the preliminary "sequence selection" phase, bone marrow oedema was not assessed because the low field MRI unit used only allows assessment of bone marrow oedema by the STIR sequence, while spectral fat saturation is not technically possible.

\section{MRI interpretation: main evaluation}

Each image set was evaluated separately for the presence or absence of MRI erosions, synovitis, and bone marrow oedema by an observer who was unaware of the findings by other modalities.

MRI bone erosions were evaluated separately in the metacarpal head and the phalangeal base of each MCP joint and in each wrist bone. Synovitis in MCP joints was evaluated for the whole joint, while the wrist joint was 
Table 2 Preliminary MRI evaluation*: detection of bone erosions and synovitis of low field spin echo and gradient echo MRI with high field spin echo MRI as standard reference

\begin{tabular}{|c|c|c|c|c|c|c|}
\hline & \multicolumn{6}{|c|}{ Low field MRI } \\
\hline & \multicolumn{2}{|c|}{ Sensitivity (\%) } & \multicolumn{2}{|c|}{ Specificity (\%) } & \multicolumn{2}{|c|}{ Accuracy (\%) } \\
\hline & $\mathrm{T}_{1}$-SE & $\mathrm{T}_{1}$-GE & $\mathrm{T}_{1}-\mathrm{SE}$ & $\mathrm{T}_{1}$-GE & $\mathrm{T}_{1}$-SE & $\mathrm{T}_{1}$-GE \\
\hline Wrist erosions & 56 & 87 & 95 & 90 & 86 & 89 \\
\hline MCP erosions & 35 & 94 & 99 & 96 & 92 & 96 \\
\hline Wrist synovitis & 85 & 92 & 86 & 71 & 85 & 85 \\
\hline MCP synovitis & 65 & 81 & 97 & 73 & 79 & 78 \\
\hline
\end{tabular}

*Based on assessment of 10 patients and 10 controls.

$\mathrm{T}_{1}-\mathrm{SE}, \mathrm{T}_{1}$ weighted spin echo; $\mathrm{T}_{1}-\mathrm{GE}, \mathrm{T}_{1}$ weighted gradient echo.

divided into three regions: the radioulnar area, the radiocarpal area, and the intercarpal-carpometacarpal area. Finally, bone marrow oedema was evaluated separately in the metacarpal head and the phalangeal base of each MCP joint and in each wrist bone. MRI bone erosions, synovitis, and bone marrow oedema were defined according to the latest OMERACT recommendations. ${ }^{20}$

\section{Statistical analysis}

Agreement was calculated as sensitivity, specificity, and accuracy. Intraobserver agreement was expressed as an intraclass correlation coefficient ${ }^{24}$ (ICC, two-way mixed effects model with absolute agreement) using the Statistical Package for the Social Sciences for Windows, version 12 (SPSS, Chicago, Illinois). For the ICC calculations, $\mathrm{p}<0.05$ was considered significant.

\section{RESULTS}

Table 1 shows the characteristics of the patients.
The results of the evaluations of the MR images are divided into two parts-that is, the results from the preliminary MR image evaluation and the results from the main MR image evaluation.

\section{Results of the preliminary MR image evaluation}

Below, the performance of spin echo and gradient echo imaging on the low field unit, with high field MRI as standard reference, is described.

For destructive joint changes, the sensitivity of the $T_{1}$ weighted gradient echo images $\left(\mathrm{T}_{1}\right.$-GE) was markedly higher than the sensitivity of the $T_{1}$ weighted spin echo images $\left(T_{1}\right.$ SE) (table 2). This gain in sensitivity was obtained without compromising the specificity and accuracy.

When synovitis was evaluated, the sensitivity was moderately higher on $\mathrm{T}_{1}$-GE images than on $\mathrm{T}_{1}$-SE images, but this was at the expense of slightly lower specificity, but not accuracy (table 2).

Based on the findings of an overall higher accuracy of the GE sequence in the preliminary study, it was decided to do all

Table 3 Bone erosions and bone marrow oedema in wrist and MCP joint bones. Performance of low field MRI and $x$ ray examination when high field MRI is considered as standard reference

\begin{tabular}{|c|c|c|c|c|c|c|c|c|c|c|c|c|c|c|c|c|}
\hline \multirow[b]{3}{*}{ Examined bones ( $n$ ) } & \multicolumn{10}{|c|}{ Bone erosions } & \multicolumn{6}{|c|}{ Bone marrow oedema } \\
\hline & \multirow{2}{*}{$\begin{array}{l}\text { High } \\
\text { field } \\
\text { MRI } \\
\\
\text { (No) }\end{array}$} & \multicolumn{5}{|c|}{ Low field MRI } & \multicolumn{4}{|c|}{$x$ Ray examination } & \multirow{2}{*}{$\begin{array}{l}\begin{array}{l}\text { High } \\
\text { field MRI }\end{array} \\
\text { (No) }\end{array}$} & \multicolumn{5}{|c|}{ Low field MRI } \\
\hline & & (No) & $\begin{array}{l}\text { Sens } \\
(\%)\end{array}$ & $\begin{array}{l}\text { Spec } \\
(\%)\end{array}$ & $\begin{array}{l}\text { Acc } \\
(\%)\end{array}$ & $\mathrm{ICC}^{*}$ & (No) & $\begin{array}{l}\text { Sens } \\
(\%)\end{array}$ & $\begin{array}{l}\text { Spec } \\
(\%)\end{array}$ & $\begin{array}{l}\text { Acc } \\
\text { (\%) }\end{array}$ & & (No) & $\begin{array}{l}\text { Sens } \\
(\%)\end{array}$ & $\begin{array}{l}\text { Spec } \\
(\%)\end{array}$ & $\begin{array}{l}\text { Acc } \\
(\%)\end{array}$ & $\mathrm{ICC}^{* *}$ \\
\hline 2nd MCP prox. (57) & 21 & 22 & 100 & 97 & 98 & 0.991 & 8 & 32 & 100 & 74 & 13 & 9 & 62 & 98 & 89 & 0.853 \\
\hline 2nd MCP dist. (57) & 9 & 14 & 100 & 90 & 91 & 0.965 & 7 & 50 & 96 & 89 & 5 & 0 & 0 & 100 & 91 & $\dagger$ \\
\hline 3rd MCP prox. (57) & 18 & 21 & 100 & 92 & 95 & 0.947 & 5 & 26 & 100 & 78 & 10 & 3 & 30 & 100 & 88 & 0.604 \\
\hline 3rd MCP dist. (57) & 8 & 8 & 100 & 100 & 100 & 0.951 & 4 & 38 & 98 & 91 & 2 & 1 & 50 & 100 & 98 & 0.665 \\
\hline 4th MCP prox. (57) & 11 & 11 & 91 & 98 & 96 & 0.907 & 3 & 23 & 100 & 85 & 3 & 2 & 33 & 98 & 95 & $0.264 \ddagger$ \\
\hline 4th MCP dist. (57) & 4 & 4 & 75 & 98 & 96 & 0.915 & 4 & 60 & 98 & 95 & 1 & 0 & 0 & 100 & 98 & $\dagger$ \\
\hline 5th MCP prox. (57) & 12 & 12 & 92 & 98 & 96 & 0.969 & 4 & 31 & 100 & 86 & 7 & 2 & 29 & 100 & 91 & 0.591 \\
\hline 5th MCP dist. (57) & 6 & 5 & 83 & 100 & 98 & 0.966 & 4 & 67 & 100 & 97 & 2 & 0 & 0 & 100 & 96 & $\dagger$ \\
\hline Radius (65) & 17 & 19 & 100 & 96 & 97 & 0.884 & 4 & 24 & 100 & 80 & 5 & 1 & 20 & 100 & 94 & 0.660 \\
\hline Ulna (65) & 28 & 28 & 96 & 97 & 97 & 0.940 & 9 & 29 & 97 & 89 & 6 & 4 & 50 & 98 & 94 & 0.350 \\
\hline Scaphoid (65) & 21 & 19 & 81 & 95 & 91 & 0.947 & 12 & 57 & 100 & 86 & 10 & 5 & 40 & 98 & 89 & 0.682 \\
\hline Lunate (65) & 23 & 24 & 96 & 95 & 95 & 0.968 & 8 & 35 & 100 & 77 & 8 & 4 & 38 & 98 & 91 & $0.139 \ddagger$ \\
\hline Triquetrum (65) & 20 & 24 & 90 & 87 & 88 & 0.957 & 5 & 20 & 98 & 74 & 2 & 2 & 50 & 98 & 97 & 0.564 \\
\hline Pisiform (65) & 5 & 7 & 100 & 97 & 97 & 0.819 & 6 & 60 & 95 & 92 & 1 & 0 & 0 & 98 & 98 & $0.000 \ddagger$ \\
\hline Trapezium (65) & 16 & 20 & 100 & 92 & 94 & 0.974 & 7 & 25 & 94 & 77 & 3 & 1 & 33 & 100 & 97 & 0.899 \\
\hline Trapezoid (65) & 11 & 16 & 91 & 89 & 89 & 0.976 & 4 & 36 & 100 & 89 & 2 & 1 & 50 & 100 & 98 & 0.663 \\
\hline Capitate (65) & 32 & 40 & 100 & 76 & 88 & 0.955 & 7 & 22 & 100 & 62 & 6 & 6 & 67 & 97 & 94 & 0.496 \\
\hline Hamate (65) & 13 & 21 & 100 & 85 & 88 & 0.959 & 7 & 46 & 98 & 88 & 3 & 3 & 100 & 100 & 100 & 0.959 \\
\hline 1st Base (65) & 9 & 13 & 100 & 93 & 94 & 0.873 & 2 & 22 & 100 & 89 & 2 & 1 & 50 & 100 & 98 & 0.544 \\
\hline 2nd Base (65) & 14 & 17 & 93 & 92 & 92 & 0.935 & 3 & 21 & 100 & 83 & 3 & 3 & 67 & 98 & 97 & 0.907 \\
\hline 3rd Base (65) & 9 & 11 & 89 & 95 & 94 & 0.972 & 3 & 33 & 100 & 91 & 3 & 4 & 100 & 98 & 98 & 0.890 \\
\hline 4th Base (65) & 9 & 10 & 100 & 98 & 98 & 0.833 & 2 & 22 & 100 & 89 & 4 & 2 & 50 & 100 & 97 & 0.875 \\
\hline 5th Base (65) & 2 & 4 & 100 & 97 & 97 & 0.929 & 5 & 0 & 92 & 89 & 1 & 1 & 0 & 98 & 97 & $-0.013 \ddagger$ \\
\hline Total (1431) & 318 & 370 & 94 & 93 & 94 & 0.936 & 123 & 33 & 98 & 83 & 102 & 55 & 39 & 99 & 95 & - \\
\hline
\end{tabular}



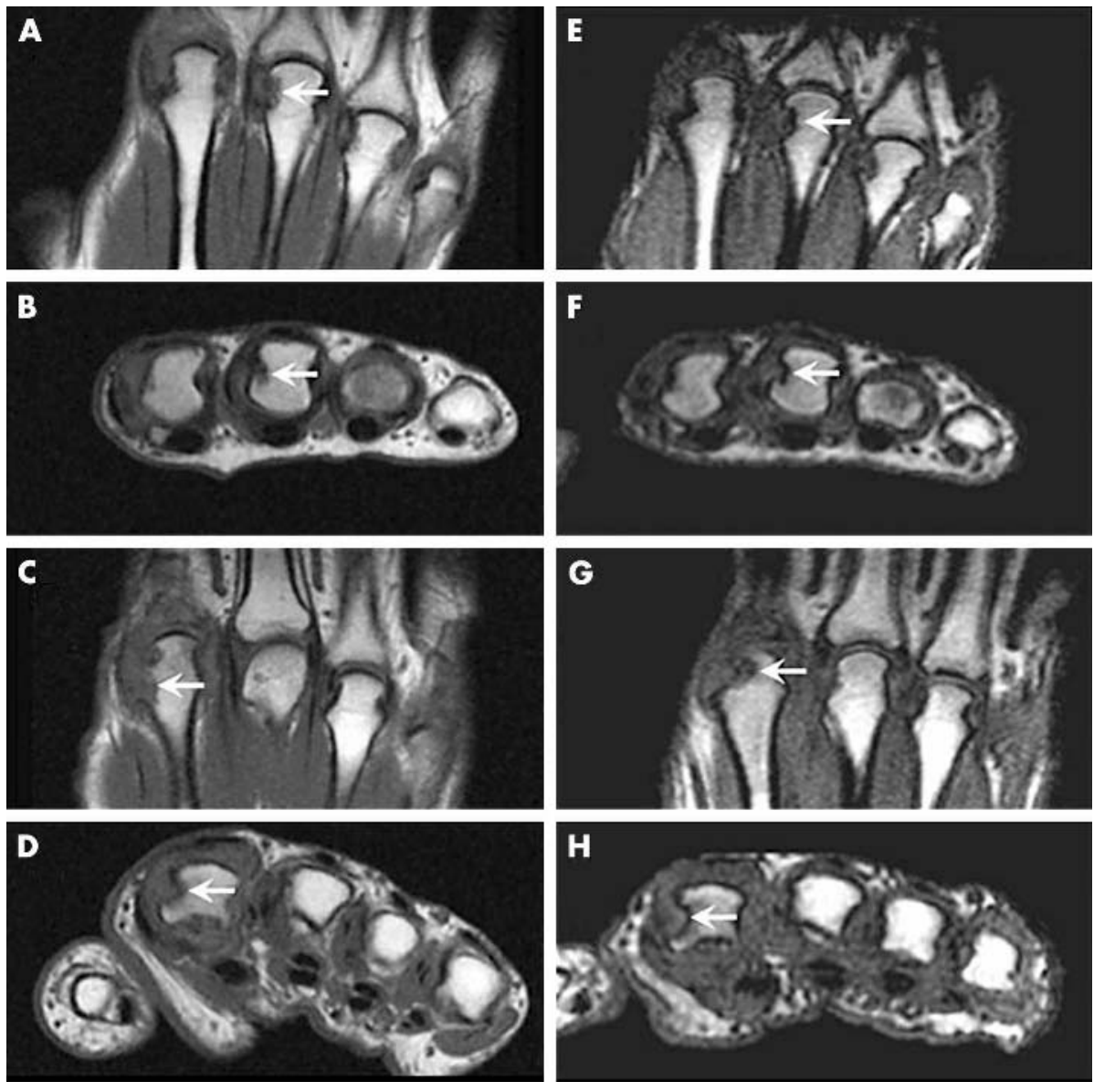

Figure 1 Erosions in RA MCP joint bones visualised by high field and low field MRI. High field (A, B, C, D) and low field $(E, F, G, H)$ coronal $(A, C, E$, $G)$ and axial $(B, D, F, H)$ images of the 2nd-5th MCP joints. On high field MRI as well as low field MRI, an erosion (OMERACT grade 2) (arrows) is depicted in the 3rd metacarpal head $(A, B, E, F)$ in patient 1 (upper four images). In patient 2 (lower four images) an erosion (OMERACT grade 3) (arrows) is depicted in the 2nd metacarpal head at both field strengths. All displayed images were obtained before contrast injection. subsequent analyses-that is, the "main MRI evaluation", based on the data obtained on the $\mathrm{T}_{1}$-GE images of the low field MRI units.

\section{Results of the main MR image evaluation}

Bone erosions on high field and low field MRI

In total 1431 wrist and MCP joint bones were evaluated for the presence of bone erosions. High field MRI detected 318 erosions and low field MRI detected 370 erosions. The mean sensitivity, specificity, accuracy, and ICCs of low field MRI for detection and grading of erosions, when high field MRI was considered the standard reference, were found to be $94 \%$, $93 \%, 94 \%$ and $0.936(\mathrm{p}<0.005)$, respectively (table 3 , figs 1 and 2).

Bone erosions on high field MRI and $x$ ray examination

A total of 1495 bones were evaluated by $x$ ray examination and high field MRI for the presence of erosions. The number of erosions detected in the wrist and MCP joint bones was 123 and 318, respectively. The mean sensitivity, specificity, and accuracy of $x$ ray examination for detection of erosions, when high field MRI was considered the standard, was 33\%, $98 \%$, and $83 \%$, respectively (table 3 ).

\section{Synovitis on high field and low field MRI}

The numbers of areas with synovitis detected by high field MRI and low field MRI in the three wrist areas (distal radioulnar joint, radiocarpal joint, and the intercarpalcarpometacarpal area) and the MCP joints in all subjects were 172 and 164, respectively. The mean sensitivity, specificity, accuracy, and ICC of low field MRI for detection of synovitis, when high field MRI was considered the standard, were 90\%, 96\%, 94\%, and $0.923(\mathrm{p}<0.05)$, respectively (table 4 , figs 3 and 4 ).

Bone marrow oedema on high field and low field MRI As for erosions, 1431 bones were evaluated by high field MRI and low field MRI for the presence of bone marrow oedema. The numbers of bones with bone marrow oedema detected in the wrist and MCP joint bones were 102 and 55, respectively. The mean sensitivity, specificity, and accuracy of low field MRI for detection of bone marrow oedema (high field MRI was considered the standard reference) were 39\%, 99\%, and $95 \%$. The overall ICC could not be computed because some of the measurements had fewer than two non-zero values (table 3, fig 5).

\section{DISCUSSION}

The main objective of this study was to investigate whether a low field dedicated extremity MRI unit could provide information on destructive and inflammatory joint changes equivalent to that of a standard high field MRI unit.

A most pertinent but often difficult question to answer when testing a new method is which reference method the new method should be tested against. In this study we chose to use high field MRI as the standard reference because most published MRI data are based on this method and because findings from high field MRI have been shown to be highly correlated with histopathological and miniarthroscopic findings $\mathrm{s}^{25-28}$ and with future development of radiographic progression..$^{29-32}$

Only a few published studies directly compare different MRI systems for the detection of arthritic joint disease. These 

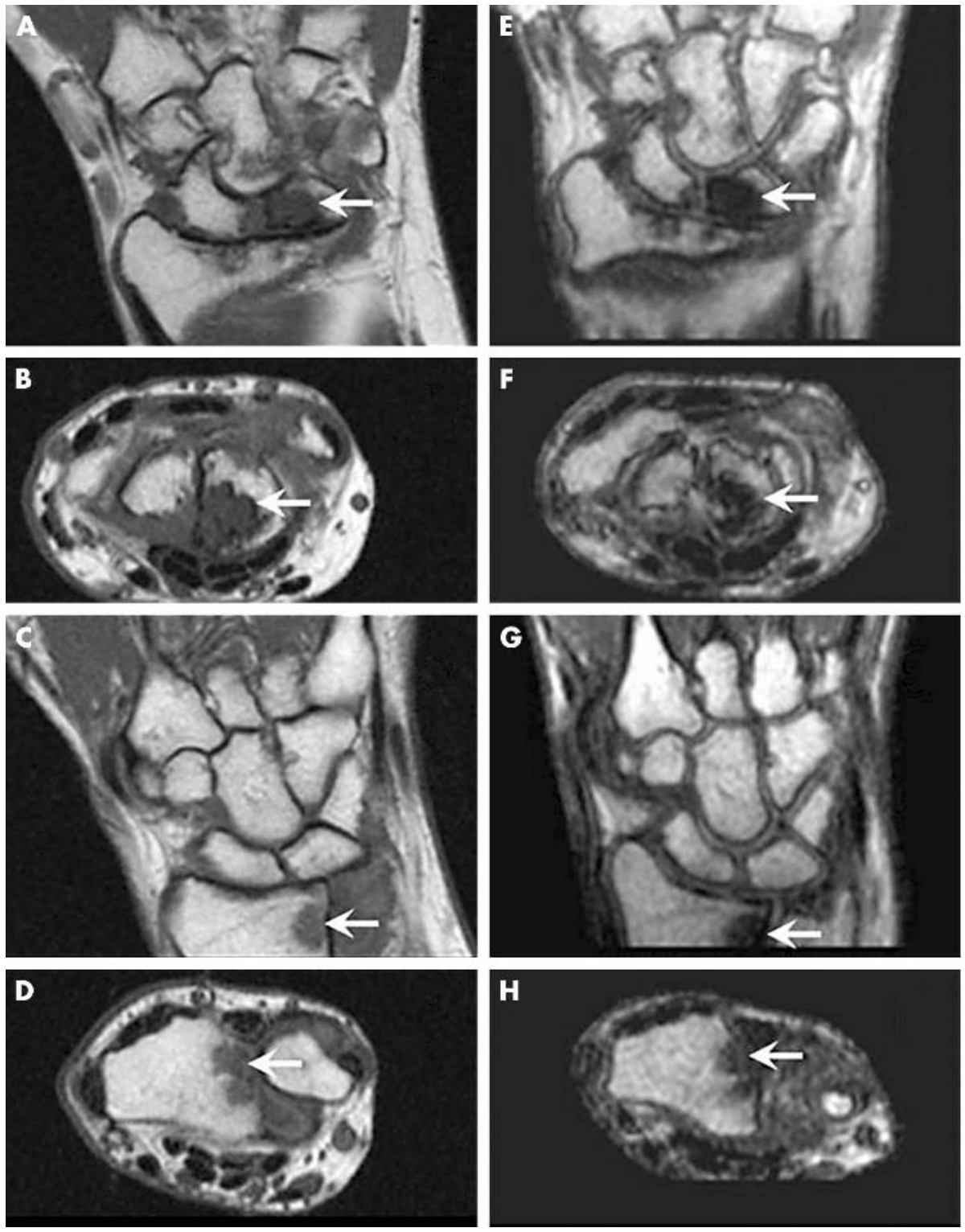
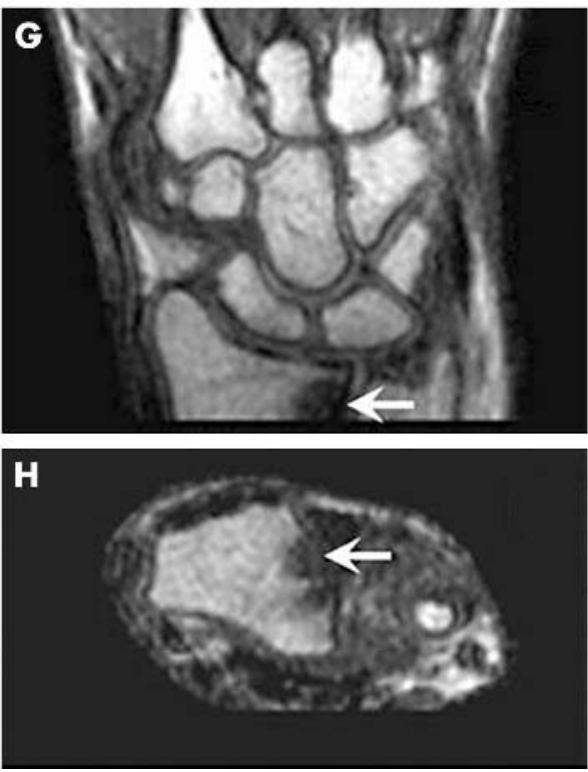

Figure 2 Erosions in RA wrist joint bones visualised by high field and low field MRI. High field $(A, B, C, D)$ and low field $(E, F, G, H)$ coronal $(A, C, E$, $G)$ and axial $(B, D, F, H)$ images of the wrist joints. On high field MRI as well as low field MRI, an erosion (OMERACT grade 5) (arrows) is depicted in the lunate $(A, B, E, F)$ in patient 1 (upper four images). In patient 2 (lower four images) an erosion (OMERACT grade

1) (arrows) is depicted in radius (C, D $G, H)$ at both field strengths. All displayed images were obtained before contrast injection.

\begin{tabular}{|c|c|c|c|c|c|c|}
\hline \multicolumn{7}{|c|}{$\begin{array}{l}\text { Table } 4 \text { Synovitis in wrist joint areas and MCP joints detected on high field and low field } \\
\text { MRI. Agreement rates of low field MRI were calculated with high field MRI as standard } \\
\text { reference }\end{array}$} \\
\hline \multirow{2}{*}{$\begin{array}{l}\text { Examined } \\
\text { joint/joint area } \\
\text { (No) }\end{array}$} & \multirow[b]{2}{*}{$\begin{array}{l}\text { High field MRI } \\
\text { (No) }\end{array}$} & \multicolumn{5}{|c|}{ Low field MRI } \\
\hline & & (No) & $\begin{array}{l}\text { Sensitivity } \\
(\%)\end{array}$ & $\begin{array}{l}\text { Specificity } \\
(\%)\end{array}$ & $\begin{array}{l}\text { Accuracy } \\
(\%)\end{array}$ & $\mathrm{ICC}^{*}$ \\
\hline 2nd MCP (57) & 30 & 27 & 87 & 96 & 91 & 0.928 \\
\hline 3rd MCP (57) & 22 & 20 & 86 & 97 & 93 & 0.908 \\
\hline 4th MCP (57) & 12 & 11 & 92 & 100 & 98 & 0.924 \\
\hline 5th MCP (57) & 15 & 14 & 87 & 98 & 95 & 0.945 \\
\hline Ra-Ul (65) & 29 & 27 & 93 & 100 & 94 & 0.945 \\
\hline Ra-Carp (65) & 37 & 39 & 97 & 89 & 94 & 0.922 \\
\hline ICMC (65) & 27 & 26 & 93 & 97 & 95 & 0.890 \\
\hline Total (423) & 172 & 164 & 90 & 96 & 94 & 0.923 \\
\hline \multicolumn{7}{|c|}{$\begin{array}{l}{ }^{*} \mathrm{p}<0.05 \text {. } \\
\mathrm{MCP} \text {, metacarpophalangeal; Ra-UI, distal radioulnar joint; Ra-Carp, radiocarpal area of wrist joint; ICMC, } \\
\text { intercarpal-carpometacarpal area of wrist joint; ICC, intraclass correlation coefficient. No, numbers of joints/joint } \\
\text { area. Note: Eight people had a limb size that precluded MRI examination of the MCP joints owing to the size of the } \\
\text { FOV. }\end{array}$} \\
\hline
\end{tabular}



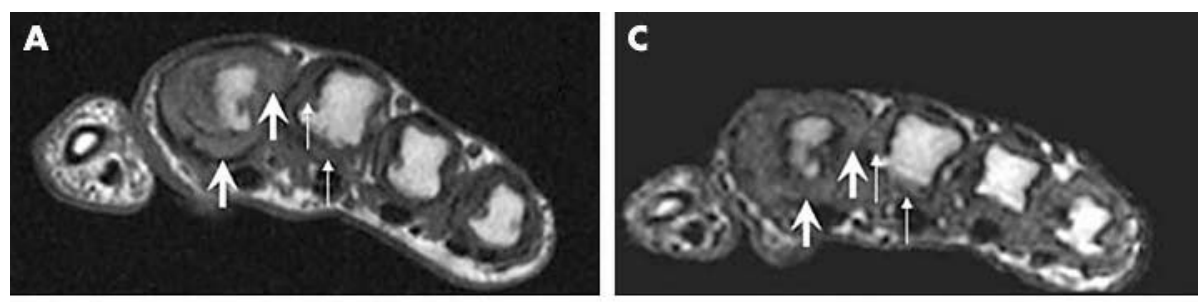

Figure 3 Synovitis in RA MCP joints visualised by high field and low field MRI. High field (A, B) and low field (C, $D)$ axial images of the 2 nd-5th $M C P$ joints before $(A, C)$ and after $(B, D)$ intravenous contrast injection. Postcontrast images show high grade synovitis (OMERACT grade 3) (arrows) in the 2nd MCP joint on high field MRI as well as on low field MRI, while low
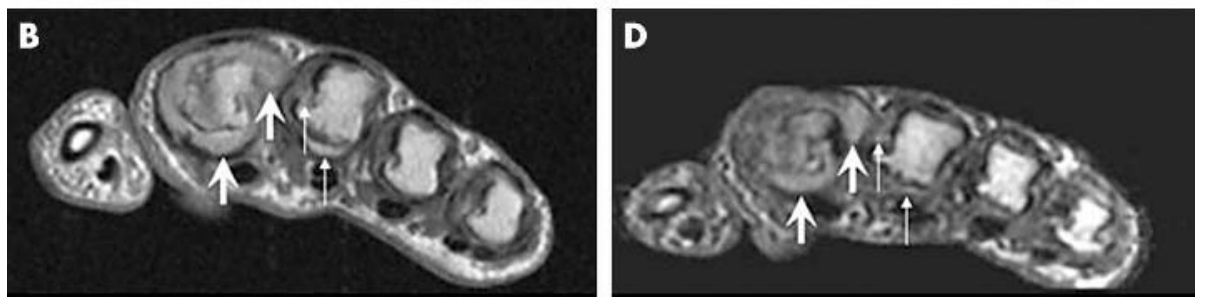
grade synovitis (OMERACT grade 1) (thin arrows) is seen in the 3rd MCP joint on images obtained at both field strengths.

studies have not incorporated any attempts to optimise the standard before starting the studies. ${ }^{13} 14$

The results of our study were divided into two major parts-namely, the results of the preliminary MR image evaluation and the results of the main study. In the preliminary image evaluation, the performance of the low field spin echo and gradient echo sequences was compared with high field MRI as the standard reference. Owing to markedly better sensitivity for bone erosions without loss of specificity, and better sensitivity for synovitis, although with
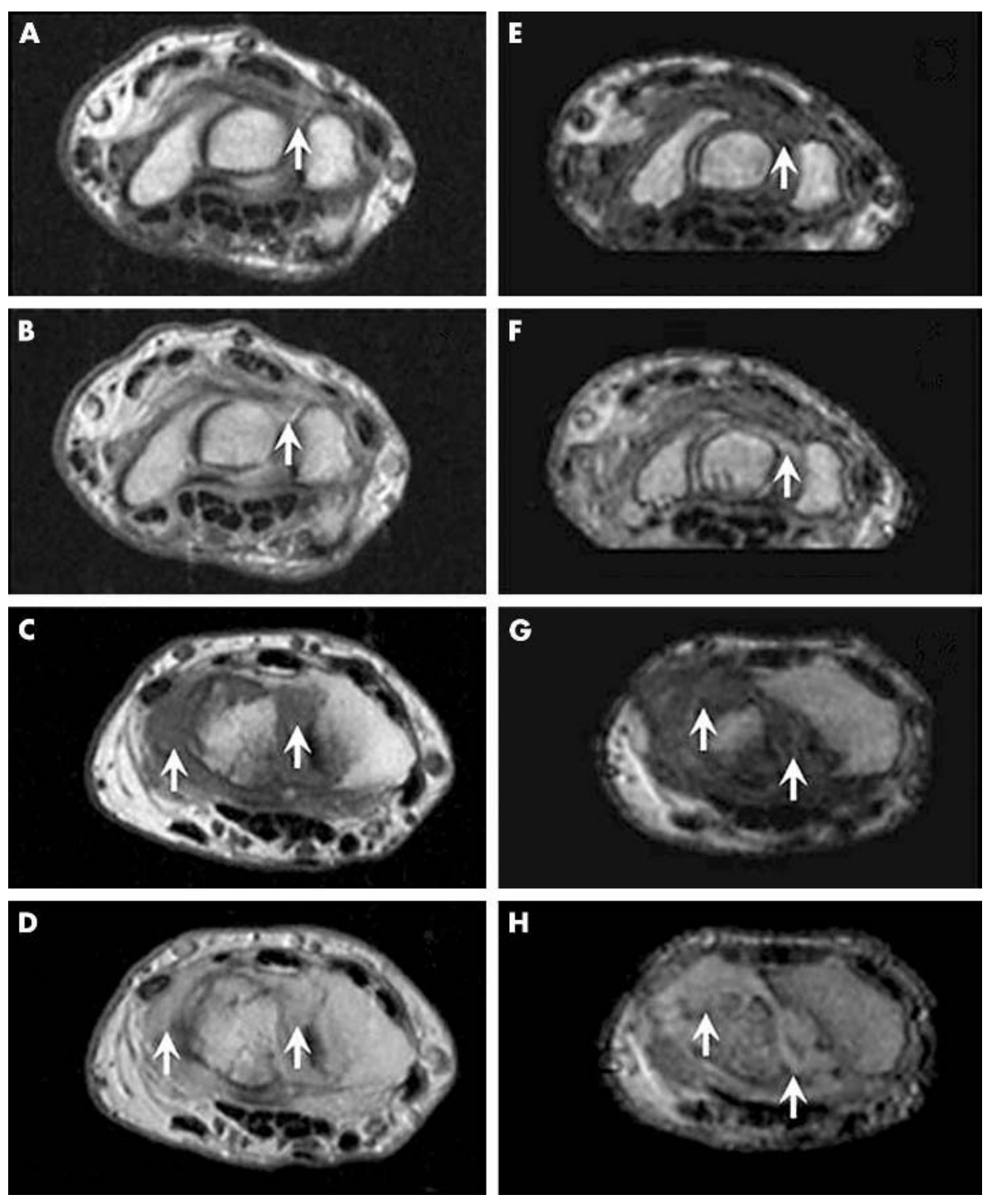
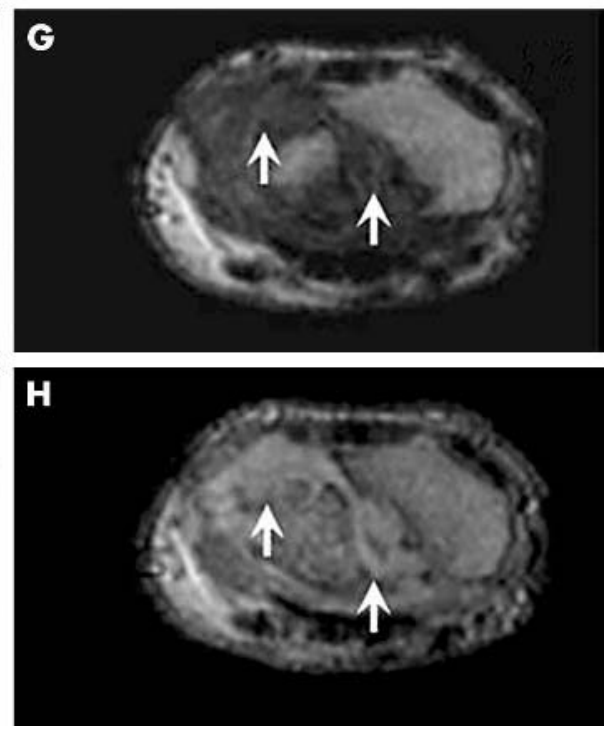

Figure 4 Synovitis in RA wrist joints visualised by high field and low field MRI. High field (A, B, C, D) and low field $(E, F, G, H)$ axial images of the wrist joint before $(A, C, E, G)$ and after $(B, D, F, H)$ intravenous contrast injection. Post-contrast images in patient 1 (upper four images) show low grade synovitis (OMERACT grade 1) (arrows) in the intercarpal area of the wrist joint on high field MRI as well as on low field MRI, while high grade synovitis (OMERACT grade 3) (arrows) is seen in the radiocarpal area of the wrist joint in patient 2 (lower four images) on images obtained at both field strengths. 

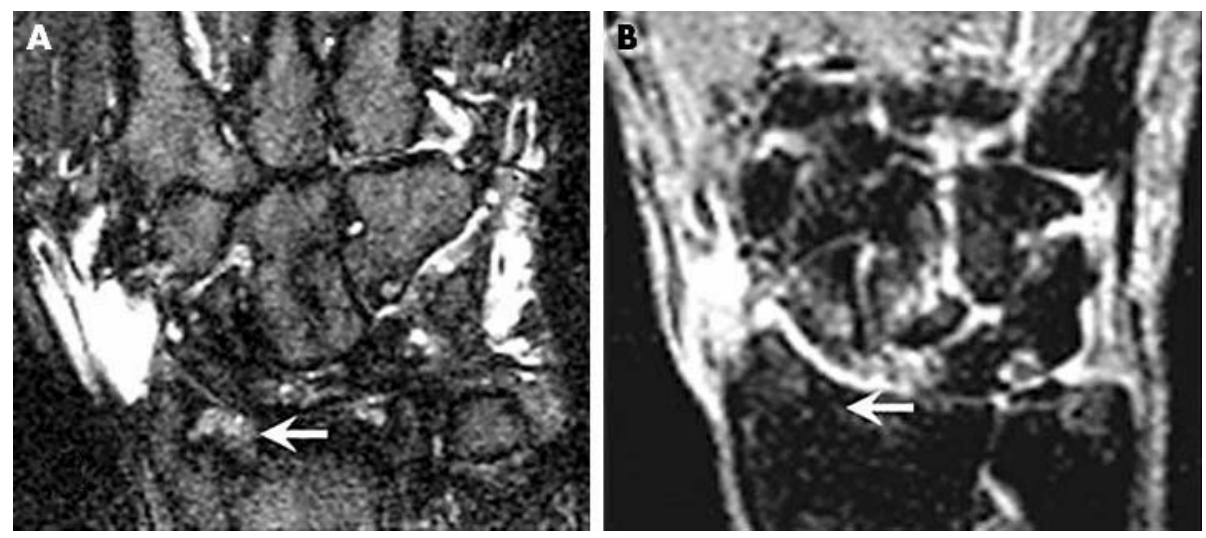

Figure 5 Bone marrow oedema in RA wrist joint bones visualised by high field and low field MRI. High field (A) and low field (B) STIR images of the wrist. On high field MRI, a low grade bone marrow oedema (OMERACT grade 1) (arrow) is seen in the distal radius. The oedema at this site is not detected on low field MRI.

a minor loss of specificity, the gradient echo sequence of the low field MRI unit was selected for the subsequent main image evaluation. Another advantage of gradient echo imaging was patient compliance, as the time needed for the MR examinations of the wrist and 2nd-5th MCP joints was about 45 minutes and 14 minutes for spin echo and gradient echo imaging, respectively.

Comparison of findings on $x$ ray examination and MRI showed that MRI detects more erosions than plain film radiography. This finding is in accordance with reports by several authors. ${ }^{73-35}$ It should be noted that plain film radiography seems to detect a higher proportion of erosions in small bones - that is, the pisiform and base of the 5th metacarpal bone compared with the larger bones (table 3). This was also reported recently by Forslind et al, who found that plain film radiography was as sensitive as MRI for the detection of bone erosions in 5th MTP joints. ${ }^{36}$

Low field MRI in the main MR image evaluation demonstrated an overall very good agreement with high field MRI for the detection of bone erosions and synovitis.

For bone erosions, the agreement rates of the low field MRI unit and the high field were very encouraging, with overall sensitivities, specificities, accuracies, and ICCs of $94 \%, 93 \%$, $94 \%$, and $0.936(\mathrm{p}<0.005)$, respectively. The findings are to some degree in accordance with data published by Savnik et al and Taouli et al. ${ }^{13}{ }^{14}$ However, Savnik's group found similar rates based on total destruction scores, but for the individual bone or joint area the agreement rates were not as high. ${ }^{13}$ Taouli et al have recently reported that high field and low field MRI are equally informative about erosive joint changes in wrists and finger joints. However, Taouli only reported erosion scores and not the results of the assessments of the individual bones. Our data show that the low field dedicated extremity MRI unit could provide information on bone erosions equivalent to that of high field MRI for the individual bones-both with respect to detection and grading.

The agreement rates for synovitis were comparable to the findings for the destructive changes. The low field MRI unit displayed overall sensitivities, specificities, accuracies, and ICCs of 90\%, 96\%, 94\%, and $0.923(\mathrm{p}<0.05)$, respectively. These findings are also comparable to previously published data, although the agreement rates were slightly lower. ${ }^{13}$ The poorer accuracy for synovitis, as well as erosions, in this study may be attributed to the lack of optimisation of the scanning sequences on the reference MRI unit. ${ }^{13}$ The study by Taouli et al also compared the synovitis scores, but their study only assessed synovitis by non-enhanced MRI in the coronal plane. The study by Taouli may suffer from a lack of sensitivity owing to the fact that the assessment of synovitis was performed on non-enhanced MR images—but on the other hand, the premises were the same for both images sets (high field and low field).

In view of our data, we conclude that with optimal MRI sequence selection, the low field dedicated extremity MRI unit can provide information on inflammatory changes (synovitis) in RA peripheral joints comparable to that obtained by contrast enhanced high field MRI, using standard 2D spin echo sequences.

The results of the comparison of the two MRI units for detection of bone marrow oedema were not as encouraging as the corresponding findings for erosions and synovitis. The overall sensitivity, specificity, and accuracy of the low field MRI unit were 39\%, 99\%, and 95\%, respectively. The ICC levels were generally low. Our conclusion was that the sensitivity for bone marrow oedema on the low field MRI unit was low. However, when bone marrow oedema was demonstrated on the low field MRI unit, the findings were almost always correct (high specificity).

The low sensitivity of the low field MRI unit for the detection of bone marrow oedema may limit the usefulness of this type of scanner in RA if bone marrow oedema is proved to be a pathological event of major prognostic significance, as has been proposed by McQueen and others. ${ }^{29}{ }^{37}$ But if bone marrow oedema is only an interim phase between synovitis and bone erosion, this may not have major impact on the usefulness of low field MRI in RA because the precursor of bone marrow oedema is generally accepted as being synovitis. The latter statement still needs to be validated in further scientific studies.

We found joint changes resembling bone erosions and synovitis in the healthy population, although only a few and only low grade changes. On the contrary, bone marrow oedema was not detected in any healthy subject. A more detailed description of these aspects has been reported elsewhere. $^{38}$

In conclusion the low field dedicated extremity MRI unit used in this study provided information on bone erosions and synovitis comparable to the information obtained by high field MRI. High overall agreement was achieved for these types of abnormalities. $x$ Ray examination displayed a markedly lower sensitivity and accuracy, though the specificity for detection of bone erosions was slightly higher than in low field MRI.

The low field MRI unit displayed high specificity but only moderate sensitivity for detection of bone marrow oedema. Further studies are needed to clarify the diagnostic and 
prognostic significance of the observed sensitivity of low field MRI for bone marrow oedema.

\section{ACKNOWLEDGEMENT}

Supported by: Amersham Health and the Danish Rheumatism Association.

\section{Authors' affiliations}

B J Ejbjerg, E Narvestad, S Jacobsen, H Thomsen, M Østergaard, Departments of Rheumatology and Department of Radiology, Copenhagen University Hospitals at Hvidovre, Rigshospitalet and Herlev, Copenhagen, Denmark

\section{REFERENCES}

1 Emery $\mathrm{P}$. The Roche Rheumatology Prize Lecture. The optimal management of early rheumatoid disease: the key to preventing disability, $\mathrm{Br} J$ Rheumatol 1994:33:765-8.

2 ACR. Guidelines for the management of rheumatoid arthritis: 2002 update. Arthritis Rheum 2002;46:328-46.

3 O'Dell JR. Treating rheumatoid arthritis early: a window of opportunity? Arthritis Rheum 2002;46:283-5.

4 Brower AC. Use of the radiograph to measure the course of rheumatoid arthritis. The gold standard versus fool's gold. Arthritis Rheum 1990;33:316-24.

5 McQueen FM, Stewart N, Crabbe J, Robinson E, Yeoman S, Tan PL, et al. Magnetic resonance imaging of the wrist in early rheumatoid arthritis reveals a high prevalence of erosions at four months after symptom onset. Ann Rheum Dis 1998;57:350-6.

6 Backhaus M, Kamradt T, Sandrock D, Loreck D, Fritz J, Wolf KJ, et al. Arthritis of the finger joints: a comprehensive approach comparing conventional radiography, scintigraphy, ultrasound, and contrast-enhanced magnetic resonance imaging. Arthritis Rheum 1999;42:1232-45.

7 Foley-Nolan D, Stack JP, Ryan M, Redmond U, Barry C, Ennis J, et al. Magnetic resonance imaging in the assessment of rheumatoid arthritis-a comparison with plain film radiographs. Br J Rheumatol 1991;30:101-6.

8 Klarlund M, Ostergaard M, Jensen KE, Madsen JL, Skjodt H, Lorenzen I, et al. Magnetic resonance imaging, radiography, and scintigraphy of the finger joints: one year follow up of patients with early arthritis. Ann Rheum Dis 2000;59:521-8.

9 Sugimoto H, Takeda A, Masuyama J, Furuse M. Early-stage rheumatoid arthritis: diagnostic accuracy of MR imaging. Radiology 1996;198:185-92.

10 Klarlund M, Ostergaard M, Gideon P, Sorensen K, Jensen KE, Lorenzen I. Wrist and finger joint MR imaging in rheumatoid arthritis. Acta Radiol 1999:40:400-9.

11 Lindegaard H, Vallo J, Horslev-Petersen K, Junker P, Ostergaard M. Low field dedicated magnetic resonance imaging in untreated rheumatoid arthritis of recent onset. Ann Rheum Dis 2001;60:770-6.

12 Crues JV, Shellock FG, Dardashti S, James TW, Troum OM. Identification of wrist and metacarpophalangeal joint erosions using a portable magnetic resonance imaging system compared to conventional radiographs. J Rheumatol 2004;31:676-85.

13 Savnik A, Malmskov H, Thomsen HS, Bretlau T, Graff LB, Nielsen H, et al. MRI of the arthritic small joints: comparison of extremity MRI $(0.2 \mathrm{~T})$ vs high-field MRI (1.5 T). Eur Radiol 2001;11:1030-8.

14 Taouli B, Zaim S, Peterfy CG, Lynch JA, Stork A, Guermazi A, et al. Rheumatoid arthritis of the hand and wrist: comparison of three imaging techniques. AJR Am J Roentgenol 2004;182:937-43.

15 Arnett FC, Edworthy SM, Bloch DA, McShane DJ, Fries JF, Cooper NS, et al. The American Rheumatism Association 1987 revised criteria for the classification of rheumatoid arthritis. Arthritis Rheum 1988;31:315-24.

16 Scott DL, van Riel PL, van der Heijde DM, Bencke AS. EULAR handbook of clinical assessments in rheumatoid arthritis (on behalf of the EULAR Comittee for International Clinical Studies including Therapeutic Trials-ESCISIT). Holland: Van Zuiden Communications BV, 2000

17 Bruce B, Fries JF. The Stanford Health Assessment Questionnaire: dimensions and practical applications. Health Qual Life Outcomes 2003;1:20.

18 Prevoo ML, van 't Hof MA, Kuper HH, van Leeuwen MA, van de Putte LB, van Riel PL. Modified disease activity scores that include twenty-eight-joint counts. Development and validation in a prospective longitudinal study of patients with rheumatoid arthritis. Arthritis Rheum 1995;38:44-8.
19 Norgaard F. Earliest roentgen changes in polyarthritis of the rheumatoid type. Continued investigations. Radiology 1969;92:299-303

20 Østergaard M, Peterfy C, Conaghan P, McQueen F, Bird P, Ejbjerg B, et al. OMERACT Rheumatoid Arthritis Magnetic Resonance Imaging Studies. core set of MRI acquisitions, joint pathology definitions and the OMERACT RA-MRI scoring system. J Rheumatol. 2003;30: 1385-6; erratum, 2004;31:198.

21 Østergaard M, Hansen M, Stoltenberg M, Lorenzen I. Quantitative assessment of the synovial membrane in the rheumatoid wrist: an easily obtained MRI score reflects the synovial volume. Br J Rheumatol 1996;35:965-71

22 Østergaard M, Gideon P, Sorensen K, Hansen M, Stoltenberg M, Henriksen $O$, et al. Scoring of synovial membrane hypertrophy and bone erosions by MR imaging in clinically active and inactive rheumatoid arthritis of the wrist. Scand J Rheumatol 1995;24:212-18.

23 Klarlund $M$, Ostergaard $M$, Lorenzen I. Finger joint synovitis in rheumatoid arthritis: quantitative assessment by magnetic resonance imaging. Rheumatology (Oxford) 1999;38:66-72.

24 Shrout PE, Fleiss JL. Intraclass correlations: uses in assessing rater reliability. Psychological Bulletin 1979;86:420-8.

25 Ostendorf B, Peters R, Dann P, Becker A, Scherer A, Wedekind F, et al. Magnetic resonance imaging and miniarthroscopy of metacarpophalangeal joints: sensitive detection of morphologic changes in rheumatoid arthritis. Arthritis Rheum 2001:44:2492-502.

26 Konig H, Sieper J, Wolf KJ. Rheumatoid arthritis: evaluation of hypervascular and fibrous pannus with dynamic MR imaging enhanced with Gd-DTPA. Radiology 1990;176:473-7.

27 Gaffney K, Cookson J, Blake D, Coumbe A, Blades S. Quantification of rheumatoid synovitis by magnetic resonance imaging. Arthritis Rheum 1995;38:1610-17.

28 Østergaard M, Stoltenberg M, Lovgreen-Nielsen P, Volck B, Sonne-Holm S, Lorenzen I. Quantification of synovistis by MRI: correlation between dynamic and static gadolinium-enhanced magnetic resonance imaging and microscopic and macroscopic signs of synovial inflammation. Magn Reson Imaging 1998; 16:743-54

29 McQueen FM, Benton N, Perry D, Crabbe J, Robinson E, Yeoman S, et al. Bone edema scored on magnetic resonance imaging scans of the dominant carpus at presentation predicts radiographic joint damage of the hands and feet six years later in patients with rheumatoid arthritis. Arthritis Rheum 2003:48:1814-27.

30 McQueen FM, Stewart N, Crabbe J, Robinson E, Yeoman S, Tan PL, et al. Magnetic resonance imaging of the wrist in early rheumatoid arthritis reveals progression of erosions despite clinical improvement. Ann Rheum Dis 1999;58:156-63.

31 Østergaard $M$, Hansen M, Stoltenberg M, Jensen KE, Szkudlarek M, Pedersen-Zbinden $B$, et al. New radiographic bone erosions in the wrists of patients with rheumatoid arthritis are detectable with magnetic resonance imaging a median of two years earlier. Arthritis Rheum 2003;48:2128-31.

32 Tanaka N, Sakahashi H, Ishii S, Sato E, Hirose K, Ishima T. Synovial membrane enhancement and bone erosion by magnetic resonance imaging for prediction of radiologic progression in patients with early rheumatoid arthritis. Rheumatol Int 2005;25:103-7.

33 Jorgensen C, Cyteval C, Anaya JM, Baron MP, Lamarque JL, Sany J. Sensitivity of magnetic resonance imaging of the wrist in very early rheumatoid arthritis. Clin Exp Rheumatol 1993;11:163-8.

34 Corvetta A, Giovagnoni A, Baldelli S, Ercolani P, Pomponio G, Luchetti MM, et al. MR imaging of rheumatoid hand lesions: comparison with conventional radiology in 31 patients. Clin Exp Rheumatol 1992;10:217-22.

35 Ejbjerg B, Narvestad E, Østergaard M. X-ray requires a magnetic resonance imaging estimated bone volume loss of $20-30 \%$ to allow certain detection of bone erosions in rheumatoid arthritis metacarpophalangeal joints [abstract]. Scand J Rheumatol 2003;32:259-60.

36 Forslind K, Johanson A, Larsson EM, Svensson B. Magnetic resonance imaging of the fifth metatarsophalangeal joint compared with conventional radiography in patients with early rheumatoid arthritis. Scand J Rheumatol 2003;32:131-7.

37 Savnik A, Malmskov H, Thomsen HS, Graff LB, Nielsen H, DanneskioldSamsoe $B$, et al. MRI of the wrist and finger joints in inflammatory joint diseases at 1-year interval: MRI features to predict bone erosions. Eur Radiol 2002; 12:1203-10.

38 Ejbjerg B, Narvestad E, Rostrup E, Szkudlarek M, Jacobsen S, Thomsen HS, et al. Magnetic resonance imaging of wrist and finger joints in healthy subjects occasionally shows changes resembling erosions and synovitis as seen in rheumatoid arthritis. Arthritis Rheum 2004;50:1097-106. 